\title{
MEDICIÓN DE ANCHO DE BANDA DE COHERENCIA Y DISPERSIÓN TEMPORAL EN LA BANDA DE 2.4 GHz EN AMBIENTES INTERIORES
}

\author{
Rolando Bettancourt O. ${ }^{1} \quad$ Héctor Carrasco E. ${ }^{1} \quad$ Rodolfo Feick L. ${ }^{1}$ \\ Recibido el 8 de abril de 2005, aceptado el 10 de julio de 2005
}

\begin{abstract}
RESUMEN
En este trabajo se presentan los resultados obtenidos de mediciones de la respuesta en frecuencia del canal en la banda de $2.4 \mathrm{GHz}$. Estas fueron realizadas en ambientes interiores representativos de entornos en que se utiliza el servicio IEEE 802.11, para enlaces fijos con diversos grados de obstrucción de enlace, diferente polarización de las antenas, y distinta distancia entre transmisor y receptor. Se obtuvieron parámetros como el Ancho de Banda de Coherencia, Retardo de Exceso Promedio y Dispersión Temporal RMS. En base a los datos empíricos se obtuvieron relaciones entre la Dispersión Temporal RMS y el Ancho de Banda de Coherencia, las que se contrastan con resultados publicados por otros autores.

Palabras clave: Canal multitrayecto, medición de canal, ancho de banda de coherencia, dispersión temporal RMS, desvanecimiento selectivo en frecuencia.

\section{ABSTRACT}

In this work we present the results obtained in the measurement of frequency response of indoor fixed-wireless channels at the $2.4 \mathrm{GHz}$ frequency band. Line of sight and obstructed links were measured for diverse polarizations and link distances. Parameters such as Coherence Bandwidth, Mean Excess Delay and RMS Delay Spread were obtained. Based on the empirical results relationships between the RMS Delay Spread and Coherence Bandwidth were obtained and contrasted with those published by other authors.
\end{abstract}

Keywords: Multipath channel, channel measurement, coherence bandwidth, RMS delay spread, frequency-selective fading.

\section{INTRODUCCIÓN}

El modelado y caracterización de canales inalámbricos es una de las líneas de investigación que ha tomado gran fuerza en el último tiempo, ya que permite conocer y simular las condiciones del medio físico sobre el cual operan diversos sistemas de comunicaciones inalámbricas.

Por su parte, los servicios basados en el protocolo IEEE802.11b, que operan en la banda no licitada de 2.4 $\mathrm{GHz}$, han tenido un crecimiento significativo en el último tiempo debido a la popularidad de las redes locales inalámbricas (WLAN), por lo que el análisis de su desempeño sobre el canal físico ha sido motivo de constante análisis y discusión [1].
En este estudio se investigan las características del canal inalámbrico mediante mediciones de respuesta en frecuencia [1]-[4]. Se analiza la banda entre 2.15 y 2.65 $\mathrm{GHz}$, en ambientes interiores típicos para servicio IEEE 802.11 b. Se realiza una campaña de medición mediante un Channel Sounder basado en un Analizador Vectorial de Redes (VNA), que permite obtener la respuesta en frecuencia del canal tanto en magnitud como en fase. A partir de los datos medidos en diversas condiciones de obstrucción o visibilidad del enlace, distancia y polarización de las antenas, se obtienen parámetros significativos que permiten cuantificar la selectividad en frecuencia del canal. A partir de la información de magnitud y fase de la respuesta del canal se obtienen el ancho de banda de coherencia [2] y la respuesta a impulso. Múltiples mediciones en un entorno de unas pocas longitudes de onda permiten obtener una estimación del Perfil de Retardo de Potencia (Power

1 Departamento de Electrónica - Universidad Técnica Federico Santa María, Av. España 1680, Casilla Postal: 110-V, Valparaíso, Chile. Email: rbett@elo.utfsm.cl,hce@elo.utfsm.cl, feick@elo.utfsm.cl 
Delay Profile), con el cual se obtiene se obtiene el Mean Excess Delay y el RMS Delay Spread [5], [6].

En la sección "Procedimiento de Medición y Descripción del Entorno Medido", se describe el equipamiento utilizado en la campaña de medición y el entorno medido. A continuación, en la sección "Calibración y Procesamiento de Datos" se describen el método de calibración y la forma de procesar las mediciones. En la siguiente sección se presentan y analizan los resultados obtenidos.

\section{PROCEDIMIENTO DE MEDICIÓN Y DESCRIPCIÓN DEL ENTORNO MEDIDO}

Las mediciones se realizaron en el plano de frecuencia utilizando un Channel Sounder basado en un Analizador Vectorial de Redes Rohde\&Schwarz ZVRE, con el que se determinó la respuesta en frecuencia compleja del canal. Un esquema del equipo de medición utilizado se describe en la figura 7 (anexo). Tanto en el receptor como en el transmisor fueron utilizadas antenas de tipo dipolo, las cuales tienen un patrón de radiación omnidireccional. Para lograr niveles de señal adecuados, se utilizaron amplificadores entre las antenas y cables de bajas pérdidas que se conectan a los puertos del VNA. En las configuraciones medidas, las separaciones máximas entre antenas fueron de entre 6 a $12 \mathrm{~m}$., en entornos con diversas situaciones de obstrucción de enlace.

Mediante un brazo giratorio de $1.3 \mathrm{~m}$ de diámetro controlado por un motor paso a paso, se implementa en forma virtual, en la etapa receptora, un arreglo de antenas dispuestas en forma de anillo. Este brazo gira en pasos discretos de $12^{\circ}$, obteniéndose la respuesta en frecuencia del canal en cada punto. Con ello se completan 30 mediciones por giro en aproximadamente cinco minutos. La separación entre cada punto de medición es de alrededor de $12 \mathrm{~cm}$, que es aproximadamente una longitud de onda para $2.4 \mathrm{GHz}$, lo cual garantiza que las mediciones obtenidas están no correlacionadas entre si [1], [7]. El sistema receptor está situado sobre un trípode de aproximadamente $1.5 \mathrm{~m}$ de altura, y la antena transmisora se encuentra a una altura de alrededor de $2 \mathrm{~m}$.

Se analizó, además, el efecto de la polarización de las antenas utilizadas en los extremos transmisor y receptor. La antena del transmisor se polarizó horizontalmente, mientras que para la antena receptora se utilizó tanto polarización vertical como horizontal. Además, se ensayaron múltiples valores de separación de antenas, para analizar el efecto de la distancia en las distintas medidas que caracterizan en comportamiento del canal.
El rango de frecuencias medido fue entre $2.15 \mathrm{GHz}$ y $2.65 \mathrm{GHz}$, en 201 muestras equiespaciadas, lo cual equivale a una resolución de $2.5 \mathrm{MHz}$. El ancho de banda total medido ( $f=500 \mathrm{MHz}$ ) determina que la máxima resolución temporal es de $2 \mathrm{~ns}$, lo cual significa que no serán distinguibles componentes de multitrayecto de la respuesta a impulso separadas por tiempos menores. El rango de tiempo posible de analizar es el inverso de la resolución en frecuencia [1], lo cual corresponde a 400 ns. Se comprobó empíricamente que en los escenarios medidos una cantidad de muestras mayor no aporta nueva información, ya que la parte relevante de la respuesta a impulso queda contenida en ese intervalo.

La campaña de medición fue realizada en el Departamento de Electrónica de la Universidad Técnica Federico Santa María, en un sector donde se encuentran oficinas y laboratorios de investigación. En la figura $7 \mathrm{se}$ encuentra un esquema del ambiente de medición. Las paredes son principalmente de concreto reforzado, lo cual permite una alta reflexión de las ondas electromagnéticas. Además, este entorno permite medir situaciones tanto de obstrucción de enlace (NLOS) como situaciones de enlace en línea vista (LOS).

Se midió situando las antenas receptora y transmisora en las ubicaciones $\mathrm{AC}, \mathrm{AD}, \mathrm{BC}, \mathrm{BD}, \mathrm{BE}$ (anexo, figura 7). Utilizando el giro del brazo, se tomó en cada una de ellas un set de 30 mediciones. Los resultados de mediciones y análisis se resumen en el anexo tabla 1. Dado que cada medición toma cinco minutos, lo cual excede ampliamente el tiempo de coherencia de un enlace con dispersores móviles (personas en movimiento) las mediciones fueron hechas en horario nocturno.

\section{CALIBRACIÓNY PROCESAMIENTO DE DATOS}

El equipo VNA permite obtener, en el plano de frecuencias, la función de transferencia compleja de una red de dos puertas. En este caso la red de dos puertas corresponde al canal inalámbrico. La ganancia compleja $S_{2 l}(f)$ medida por el equipo VNA corresponde a la combinación de la característica del canal inalámbrico que se desea medir y de otros elementos que intervienen en la medición. En términos generales, la característica $S_{2 I}(f)$ corresponde a un producto de funciones de transferencia, dada por:

$$
S_{21}(f)=H_{c}(f) H_{\text {amp }}(f) H_{\text {ant }}(f) H_{V N A}(f)
$$


donde $H_{c}(f)$ corresponde a la respuesta en frecuencia del canal que se desea medir. $H_{a n t}(\mathrm{f})$ y $H_{a m p}(f)$ corresponden a las características no planas tanto de los amplificadores como de las antenas utilizadas y $H_{V N A}(f)$ engloba la característica misma del equipo y de la respuesta en frecuencia de los cables a utilizar. Esto último se elimina realizando la correspondiente calibración del equipo con cargas adaptadas. La influencia de las características en frecuencia de las antenas y amplificadores es compensada en la etapa de procesamiento de datos, debido a que éstas son medidas en forma previa, utilizando el mismo equipo. Con esto, se puede obtener la respuesta en frecuencia del canal despejando $H_{c}(\Varangle)$ de (1). En la figura 1 se aprecian dos típicas respuestas en frecuencia (magnitud) medidas.

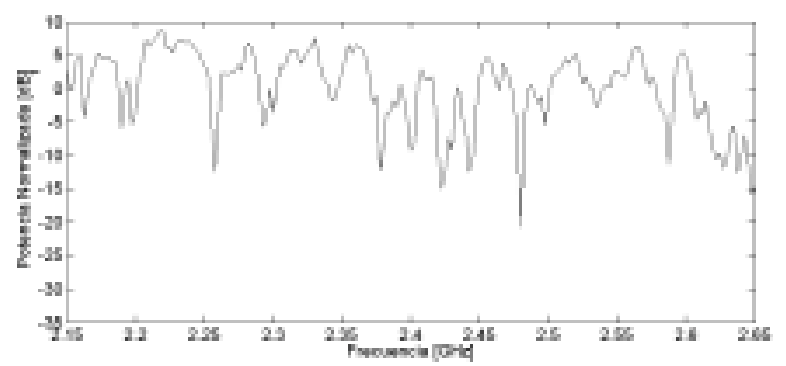

(a)

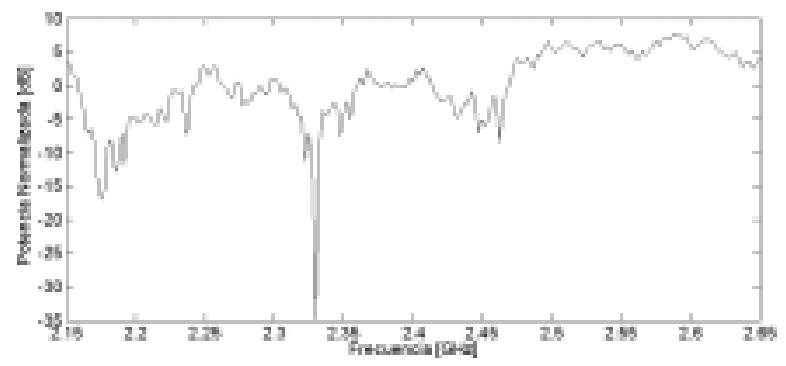

(b)

Fig. 1 Magnitud de la Respuesta en Frecuencia típica para escenarios medidos en condición NLOS (a) y LOS (b).

Mediante la respuesta en frecuencia obtenida, es posible estimar la característica temporal del canal mediante la Transformada Inversa de Fourier. Sin embargo, debido al ancho de banda finito medido, lo que se obtiene es una estimación de la respuesta a impulso del canal, $h(, t)$.

$$
h(\tau, t)=\int_{f c-\Delta f / 2}^{f c+\Delta f / 2} H(f, t) e^{j 2 \pi f \tau} d f
$$

donde, siguiendo la notación de Bello [5], $t$ corresponde al instante de tiempo en que se realizó la medición, y $\tau$ es la variable de tiempo de la duración de la respuesta. Mediante (3) se obtiene el Power Delay Profile Instantáneo, $P_{h}(\tau, t)$.

$$
P_{h}(\tau, t)=|h(\tau, t)|^{2}
$$

Asumiendo ergodicidad se obtiene el Power Delay Profile Promedio, $P_{h}(\tau)$, que en este caso es idéntico al valor instantáneo debido a que se mide en un entorno completamente estático.

$$
P_{h}(\tau)=E_{t}\left[|h(\tau, t)|^{2}\right]
$$

Esto simplemente corresponde a decir que $P_{h}(\tau, t)$ es sólo una función de $\tau$, y no del instante de medición. En la figura 2 se muestra como ejemplo $P_{h}(\tau)$ calculado a partir de las respuestas en frecuencias de la figura 1.

A partir de $P_{h}(\tau)$, se obtienen el Mean Excess Delay $\left(\tau_{\text {mean }}\right)(5)$ y el $R M S$ Delay Spread $\left(\tau_{\text {rms }}\right)(6)$, que son parámetros importantes que describen la dispersión temporal del canal, la que influye directamente en la interferencia intersimbólica (ISI) y, por lo tanto, en la máxima tasa de transmisión de un sistema sin necesidad de ecualización u otra técnica de compensación de la dispersión temporal.

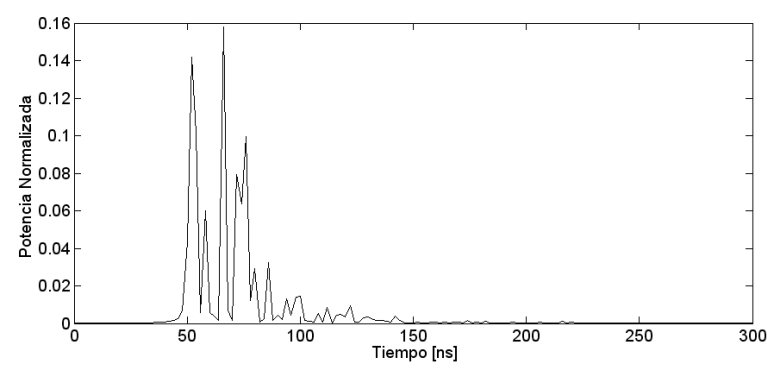

(a)

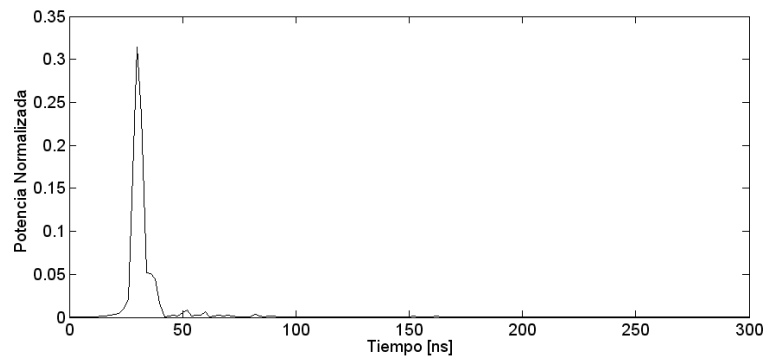

(b)

Fig. 2 Power Delay Profile obtenido mediante las Respuestas en Frecuencia anteriores. Caso (a) NLOS y (b) LOS. 


$$
\begin{gathered}
\tau_{\text {mean }}=\frac{\int_{0}^{\tau_{\max }} \tau\left|P_{h}(t, \tau)\right|^{2} d \tau}{\int_{0}^{\tau_{\max }}\left|P_{h}(t, \tau)\right|^{2} d \tau} \\
\tau_{\text {rms }}^{2}=\frac{\int_{0}^{\tau_{\max }}\left(\tau-\tau_{\text {mean }}\right)^{2}\left|P_{h}(t, \tau)\right|^{2} d \tau}{\int_{0}^{\tau_{\max }}\left|P_{h}(t, \tau)\right|^{2} d \tau}
\end{gathered}
$$

El cálculo de (2) se realizó mediante el comando ifft de Matlab ${ }^{\mathrm{TM}}$, y la estimación de $\tau_{\text {mean }}$ y $\tau_{r m s}$ se realizó discretizando (5) y (6). Antes de llevar la respuesta en frecuencia al dominio del tiempo a través de (2), ésta se multiplica por una ventana Blackman-Harris. Esto reduce el piso de ruido y las componentes espurias en $P_{h}(\tau)$ [4]. En la figura 3 se aprecia el efecto de la ventana en el promedio espacial de las 30 mediciones en caso NLOS y LOS, que corresponden a las ubicaciones A-C y B-D respectivamente, de la figura 7 .

Se calcula $\tau_{\text {mean }} \mathrm{y} \tau_{r m s}$ fijando un nivel de potencia mínimo al cual se considera significativa la estimación de $P_{h}(\tau)$. Éste se fija a $20 \mathrm{~dB}$ bajo el máximo valor de $P_{h}(\tau)$. Este nivel resulta ser un criterio razonable, siendo cercano al utilizado por otros autores, [1], [8] y permite eliminar los efectos del ruido y el error de procesamiento en la estimación de los parámetros. Este umbral mínimo determina $\tau=0$ y $\tau=\tau_{\max }$ que corresponden al primer y ultimo cruce de la potencia por dicho nivel.

Por otra parte, para cada una de las mediciones de respuesta en frecuencia se calcula la función de autocorrelación compleja (normalizada), $R(\Delta f)$ [2], definida a través de (7):

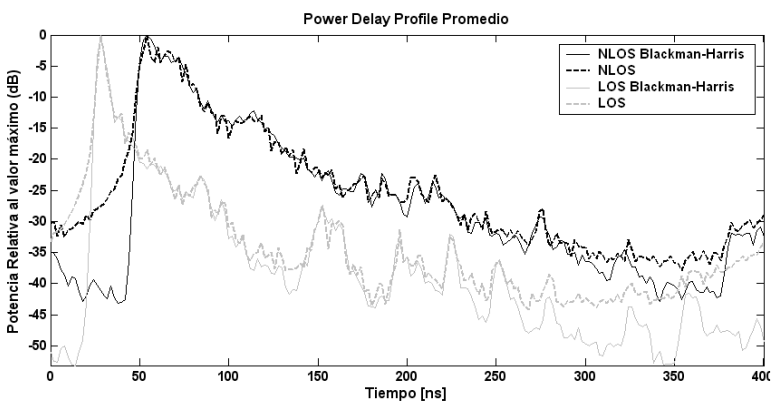

Fig. 3 Efecto de la ventana Blackman-Harris en estimación del Power Delay Profile Promedio. Caso NLOS (A-C) y LOS (B-D).

$$
R(\Delta f)=E\left[H(f)^{*} H(f+\Delta f)\right]
$$

El Ancho de Banda de Coherencia $\left(B_{C}\right)$ se define como la separación en frecuencia a la cual $|R(\Delta f)|$ (figura 4) decae por primera vez bajo un cierto nivel $c$, y es una medida estadística de la separación máxima en frecuencia a la cual se considera que el canal tiene aproximadamente la misma ganancia y que el incremento de fase es relativamente lineal [8], [9]. Se ha comprobado empíricamente y a través de modelos de simulación [9] que $B_{c}$ es inversamente proporcional a $\tau_{r m s}$. En este trabajo se calcula $B_{c}$ para un nivel de correlación $c$ de 0.9 y 0.7 .

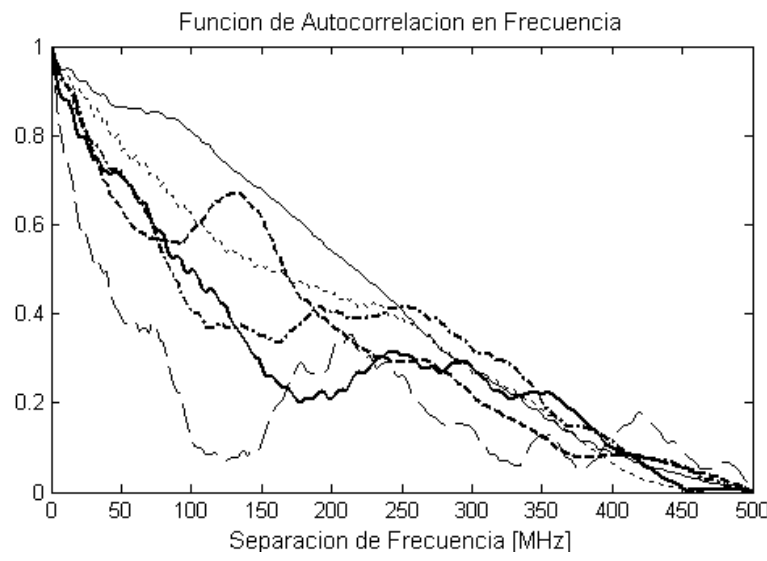

Fig. 4 Autocorrelación en Frecuencia para varias ubicaciones en condición LOS, enlace B-D.

Otros autores [7], [10] que han medido la correlación en frecuencia utilizan como método de medición dos portadoras de igual amplitud, pero separadas en frecuencia. Calculan la correlación cruzada de las envolventes de la señales a medida que la separación en frecuencia aumenta. Con esto pueden estimar una función de correlación en frecuencia de las envolventes. Los valores obtenidos utilizando uno u otro método pueden diferir.

\section{RESULTADOS OBTENIDOS}

En la tabla 1 (anexo) se resumen los resultados obtenidos en cada escenario de medición. Se calcularon los parámetros estadísticos del canal $B_{0.9} B_{0.7} \tau_{\text {mean }} \mathrm{y}$ $\tau_{r m s}$, para cada conjunto de 30 mediciones realizadas en cada una de las ubicaciones medidas. En cada ubicación, se calcularon la media y el coeficiente de variación para cada parámetro. El Coeficiente de Variación se define como: 


$$
C V=\frac{\sigma}{\bar{X}}
$$

Ésta es una medida comparativa entre la desviación estándar de los datos, $\sigma$ y la media $\bar{X}$, y resulta útil para cuantificar la variabilidad de cada parámetro, aun cuando posean valores medios distintos.

En mediciones típicas NLOS y LOS se obtienen resultados como los de las figuras 1 y 2 . Se aprecia en general que para casos LOS la magnitud de la respuesta en frecuencia sufre menos fluctuaciones bruscas y menos cambios de fase repentinos en comparación a situaciones NLOS. En el caso del Power Delay Profile, se tiene claramente que para casos LOS la respuesta está más cercana a ser un impulso, debido al haz dominante por visión directa, mientras que en el caso NLOS se aprecia que los haces de multitrayecto llegan al receptor con potencias comparables, haciendo la respuesta a impulso mucho más dispersa en tiempo. Es por esto que se comprueba que en enlaces no obstruidos (LOS) se obtiene un mayor ancho de banda de coherencia y una menor dispersión temporal que en casos NLOS.

En general se observó que para enlaces LOS el coeficiente de variación en ambos parámetros resulta ser significativamente mayor que para casos NLOS. Esto se podría atribuir a que para enlaces LOS existen unos pocos haces dominantes y consecuentemente hay más posibilidades de cancelaciones (desvanecimientos profundos) que para enlaces mas ricos en multitrayectorias como son los NLOS.

\section{RMS Delay Spread}

El RMS Delay Spread es una medida de que tan dispersa resulta ser la respuesta a impulso del canal. Se observa que los valores evidencian ser mayores para situaciones de enlace obstruido NLOS, donde no existe rayo directo. Para casos donde el rayo directo está presente, se tiene una dispersión menor. Además, ésta disminuye, en promedio, con respecto a la distancia del enlace. Lo anterior resulta ser una confirmación de la teoría. No se obtienen relaciones claras del efecto de la polarización de las antenas que sean generalizables.

En casos LOS, donde el haz directo es muy dominante, resulta difícil la estimación de $\tau_{r m s}$, debido a que la máxima resolución temporal es de $2 \mathrm{~ns}$. Esto ocasiona una respuesta más suavizada de lo que es en realidad, lo que hace que en general $\tau_{r m s}$ sea sobreestimado [4].

\section{Ancho de Banda de Coherencia}

El Ancho de Banda de Coherencia, como se esperaba, resulta ser mayor para casos LOS. Aumenta, en promedio, al disminuir la distancia transmisor-receptor, lo cual es debido a que el rayo directo se ve fortalecido con respecto a las componentes de multitrayectos originados de la reflexión y difracción en las paredes del recinto. Se puede apreciar en la figura 4 que $|R(\Delta f)|$ no decae de la misma forma, incluso tratándose de mediciones realizadas en un entorno de pocas longitudes de onda como en el que se midió. Esto corrobora la afirmación de que la forma de $|R(\Delta f)|$ depende del tipo de perfil de $P_{h}(\tau)$ [1], [9], y no simplemente de $\tau_{r m s}$.

\section{Relación entre $\mathbf{B}_{\mathbf{c}} \mathbf{y} \tau_{r m s}$}

En las figuras 5 y 6 se muestran pares $\left(\mathrm{B}_{0.9}, \tau_{r m s}\right)$ y $\left(\mathrm{B}_{0.7}, \tau_{r m s}\right)$ respectivamente, correspondiente a cada una de las mediciones efectuadas, sin distinción de condiciones del enlace. No se separaron por condición de enlace, debido a que se desea obtener una característica aplicable en general. Se aprecia claramente la relación de proporcionalidad inversa que existe entre ellos. Esta proporcionalidad existente entre estos dos parámetros se puede ajustar mediante una hipérbola, de la forma:

$$
B_{c} \approx \frac{1}{\alpha \tau_{r m s}}
$$

Esto ya se ha predicho en la literatura [8], [9]. La constante de proporcionalidad $\alpha$ se reporta del orden de las decenas para $\mathrm{B}_{0.7}[9]$. Se realiza un ajuste de los datos a (9) mediante cuadrados mínimos, obteniéndose $\alpha=16.9$ para $B_{0.9}$ y $\alpha=4.49$ para $B_{0.7}$

Se puede apreciar que el ajuste (9) para $\mathrm{B}_{0.9}$ es muy bueno, pero resulta más errático para valores pequeños de $\tau_{r m s}$. Esto se debe a que en estos casos, la estimación de $\tau_{r m s}$ está más susceptible a errores debido a que $P_{h}(\tau)$ se acerca al límite de la resolución temporal.

Para el caso de $B_{0.7}$ la tendencia es clara, pero el ajuste a (9) no es tan bueno como para $B_{0.9}$. Se puede especular que la relación (9) es en general más precisa para $B_{0.9}$ porque la estimación de la autocorrelación es más exacta para diferencias de frecuencia pequeñas, pues en tal caso se cuenta con más puntos para la estimación. Esto sugiere que es más eficaz estimar $\tau_{r m s}$ a partir de $B_{0.9}$ que a partir de $B_{0.7}$ 


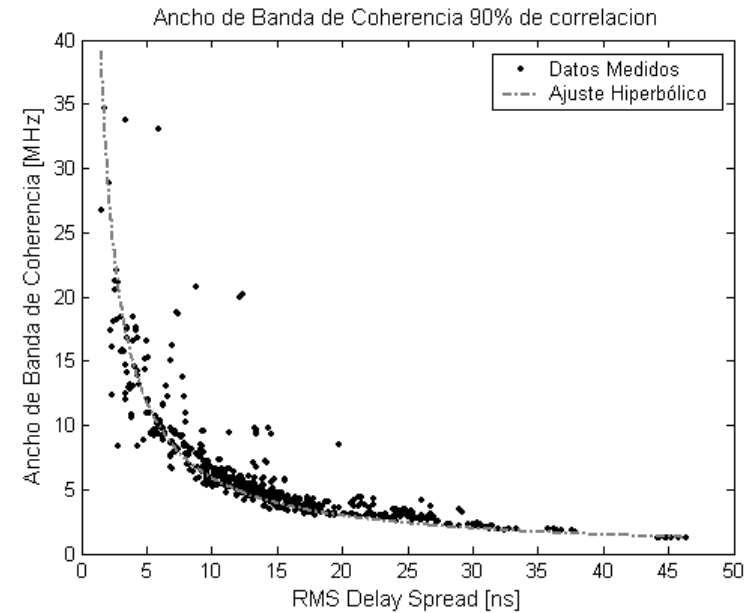

Fig. 5 Ancho de Banda de Coherencia para un nivel de 0.9 , con respecto a RMS Delay Spread.

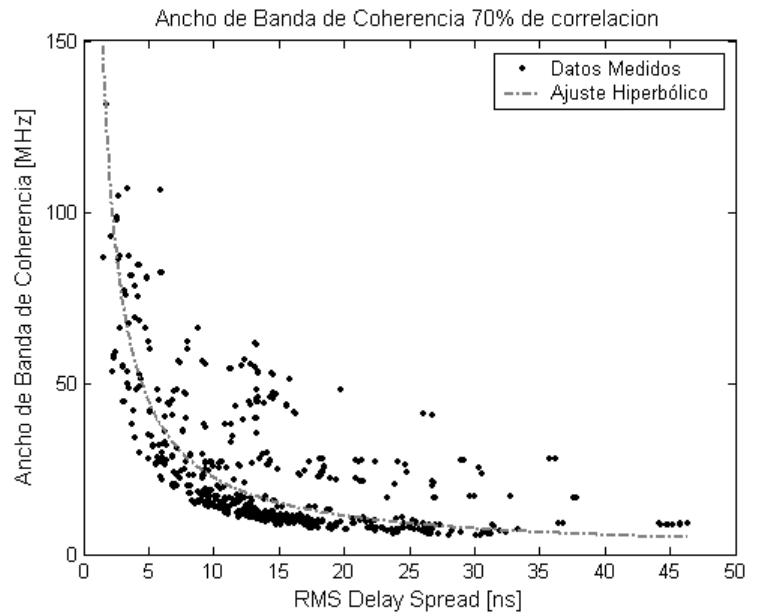

Fig. 6 Ancho de Banda de Coherencia para un nivel de 0.7 , con respecto a RMS Delay Spread.

Si se examina la figura 4 se puede notar que el comportamiento de $|R(\Delta f)|$ decae de forma relativamente suave y monótona hasta un nivel de correlación de $0.9 \mathrm{o}$ 0.85 , pero para niveles de correlación menores, la forma de la función puede sufrir cambios abruptos. Esto indica incluso que $|R(\Delta f)|$ sería más difícil de modelar en forma teórica, ya que debido al tipo de decaimiento se podría ajustar un modelo simple que aproxime teóricamente una curva simple que sea razonablemente cercana para valores de correlación de más de 0.85 , por ejemplo, pero sería más complicado buscar un modelo que reproduzca grados de correlación menores.

\section{CONCLUSIONES}

Mediciones de propagación electromagnética en el plano de la frecuencia fueron efectuadas mediante un Vector
Network Analyzer en la banda de $2.15 \mathrm{GHz}$ a $2.65 \mathrm{GHz}$. Se realizaron los procedimientos de calibración correspondientes, además de una etapa de procesamiento de datos que busca eliminar al máximo el error de estimación.

El objetivo de las mediciones fue determinar el efecto de distintas condiciones de enlace sobre el Ancho de Banda de Coherencia del canal y la dispersión temporal, caracterizada por $\tau_{\text {mean }} \mathrm{y} \tau_{r m s}$. Las variables a considerar al tomar las mediciones fueron la distancia transmisorreceptor, la polarización de las antenas y el grado de obstrucción del enlace.

Las observaciones cualitativas tanto de la Respuesta en Frecuencia y del Power Delay Profile, además de los parámetros calculados concuerdan con los supuestos, en cuanto a que $\tau_{r m s}$ y $B_{0.9}$ cumplen una relación del tipo dado en (9). Modelos de más grados de libertad resultan poco prácticos. Los resultados validan, además, en buena medida la relación de proporcionalidad inversa entre $\tau_{r m s}$ y $B_{c}$, aunque esto es mucho más exacto y confiable para $B_{0.9}$ que para $B_{0.7}$

Futuras campañas de medición resultan necesarias para comprobar los resultados en otros entornos, para poder generalizar las afirmaciones presentadas en este trabajo.

\section{AGRADECIMIENTOS}

Los autores agradecen a la Dirección General de Investigación y Postgrado de la Universidad Técnica Federico Santa María su fundamental apoyo al desarrollo de este trabajo, a través del Proyecto Interno 230322, "Modelado Estadístico de Canales Inalámbricos en las Bandas de 2.4 y $3.5 \mathrm{GHz}$ ".

\section{REFERENCIAS}

[1] G.J. Janssen, P.A. Stigter and R. Prasad. "Wideband indoor channel measurements and BER analysis of frequency selective multipath channels at 2.4, 4.75 and 11.5 GHz". IEEE Trans. Commun. Vol. 44, pp. 1272-1288. October 1996.

[2] S.J. Howard and K. Pahlavan. "Autoregresive Modeling of Wide-Band Indoor Radio Propagation". IEEE Trans. Commun. Vol. 40, pp.1540-1552. September 1992.

[3] P.F. Smulders, A.G. Wagemans. "FrequencyDomain Measurement of the Millimetre Wave Indoor Radio Channel". IEEE Trans. Instrum. Measurt. Vol. 44, pp. 1017-1022. December 1995. 
[4] M. Sánchez, M. García. "RMS Delay and Coherence Bandwidth Measurements in Indoor Radio Channels in the UHF Band". IEEE Trans. On Veh. Techn. Vol. 50. N ${ }^{\circ}$ 2. March 2001.

[5] P.A. Bello. "Characterization of randomly timevariant linear channels". IEEE Trans. Commun. Syst. Vol. CS-11, pp. 360-393. December 1963.

[6] A.A. Saleh and R.A. Valenzuela. "A statistical model for indoor multipath propagation", IEEE J. Select. Areas Commun. Vol. SAC-5, pp. 128-137. February 1987.

[7] A.F. Abouraddy, S.M. Elnoubi. "Statistical Modeling of the Indoor Radio Channel at $10 \mathrm{GHz}$ Through Propagation Measurements, Part I:
Narrow-Band Measurements and Modeling". IEEE Trans. On Veh. Techn. Vol. 49. $\mathrm{N}^{\mathrm{o}}$ 5. September 2000.

[8] T.S. Rappaport. "Wireless Communications: Principles and Practice". Prentice Hall PTR, Upper Saddle River. N. J., USA. $2^{\text {nd }}$ edition. 2002.

[9] A.F. Molisch. "Wideband Wireless Digital Communications". Prentice Hall PTR, Upper Saddle River. NJ, USA. 2001.

[10] M. Sánchez, A.M. Hammoudeh, E. Grindrod, J.P. Kermoal. "Coherence Bandwidth Characterization in an Urban Microcell at $62.4 \mathrm{GHz}$ ". IEEE Trans. On Veh. Techn. Vol. 49. № 2. March 2000.
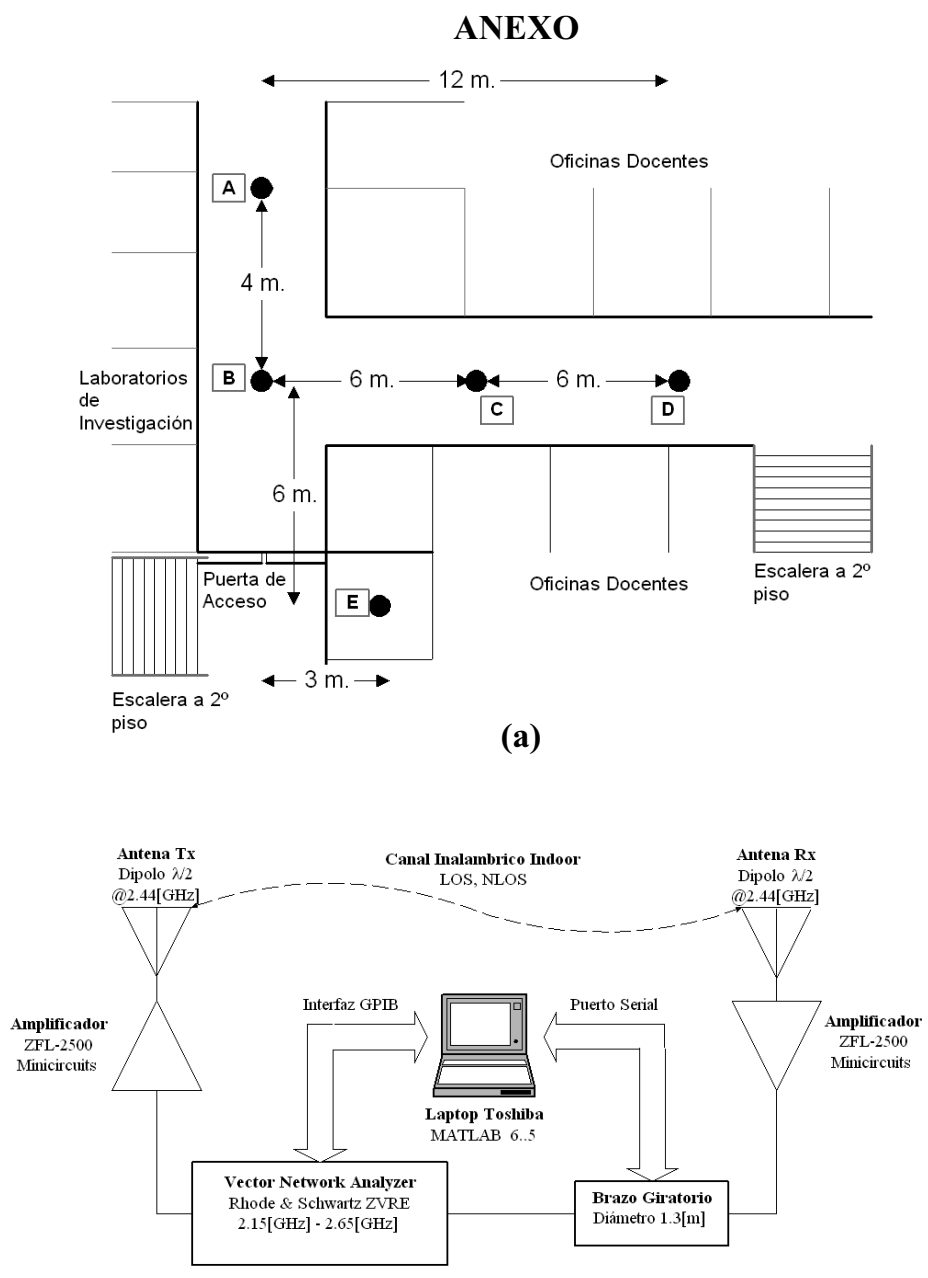

(b)

Fig. 7 (a) Entorno Medido (Departamento de Electrónica Universidad Técnica Federico Santa María, Tercer Piso). (b) Esquema de medición. 
Tabla 1 Resumen de los resultados obtenidos en cada uno de los puntos medidos.

\begin{tabular}{|c|c|c|c|c|c|c|c|}
\hline \multirow[t]{2}{*}{ Enlace } & \multirow{2}{*}{$\begin{array}{c}\text { Polarización } \\
\text { Antenas }\end{array}$} & \multicolumn{2}{|c|}{$\tau_{\mathrm{rms}} \quad[\mathrm{ns}]$} & \multicolumn{2}{|c|}{$\mathrm{B}_{0.9} \quad[\mathrm{MHz}]$} & \multicolumn{2}{|c|}{$\mathrm{B}_{0.7} \quad[\mathrm{MHz}]$} \\
\hline & & Media & C.V. & Media & C.V. & Media & C.V. \\
\hline $\begin{array}{l}\text { NLOS } \\
\text { Enlace Pasillo-Pasillo (A-C) } \\
12 \mathrm{~m}\end{array}$ & $\begin{array}{l}\text { Horizontal- } \\
\text { Horizontal }\end{array}$ & 22.09 & 0.17 & 3.30 & 0.15 & 9.08 & 0.22 \\
\hline $\begin{array}{l}\text { NLOS } \\
\text { Enlace Pasillo-Pasillo (A-C) } \\
12 \mathrm{~m}\end{array}$ & $\begin{array}{c}\text { Horizontal- } \\
\text { Vertical }\end{array}$ & 18.51 & 0.17 & 3.51 & 0.17 & 9.37 & 0.25 \\
\hline $\begin{array}{l}\text { NLOS } \\
\text { Enlace Pasillo-Pasillo (A-D) } \\
6 \mathrm{~m}\end{array}$ & $\begin{array}{c}\text { Horizontal- } \\
\text { Horizontal }\end{array}$ & 10.81 & 0.14 & 5.92 & 0.14 & 16.00 & 0.26 \\
\hline $\begin{array}{l}\text { NLOS } \\
\text { Enlace Pasillo-Pasillo (A-D) } \\
6 \mathrm{~m}\end{array}$ & $\begin{array}{l}\text { Horizontal- } \\
\text { Vertical }\end{array}$ & 13.10 & 0.16 & 5.01 & 0.17 & 12.25 & 0.25 \\
\hline $\begin{array}{l}\text { LOS } \\
\text { Enlace Pasillo-Pasillo (B-D) } \\
12 \mathrm{~m}\end{array}$ & $\begin{array}{c}\text { Horizontal- } \\
\text { Horizontal }\end{array}$ & 25.29 & 0.41 & 4.17 & 0.76 & 33.11 & 0.64 \\
\hline $\begin{array}{l}\text { LOS } \\
\text { Enlace Pasillo-Pasillo (B-D) } \\
12 \mathrm{~m}\end{array}$ & $\begin{array}{c}\text { Horizontal- } \\
\text { Vertical }\end{array}$ & 14.51 & 0.78 & 9.9 & 0.79 & 43.93 & 0.65 \\
\hline $\begin{array}{l}\text { LOS } \\
\text { Enlace Pasillo-Pasillo (B-C) } \\
6 \mathrm{~m}\end{array}$ & $\begin{array}{l}\text { Horizontal- } \\
\text { Horizontal }\end{array}$ & 12.21 & 0.38 & 8.67 & 0.60 & 44.83 & 0.31 \\
\hline $\begin{array}{l}\text { LOS } \\
\text { Enlace Pasillo-Pasillo (B-C) } \\
6 \mathrm{~m}\end{array}$ & $\begin{array}{c}\text { Horizontal- } \\
\text { Vertical }\end{array}$ & 6.32 & 0.45 & 10.72 & 0.34 & 51.42 & 0.49 \\
\hline $\begin{array}{l}\text { NLOS } \\
\text { Enlace Pasillo-Oficina (B-E) } \\
\text { Puertas Cerradas }\end{array}$ & $\begin{array}{c}\text { Horizontal- } \\
\text { Horizontal }\end{array}$ & 7.84 & 0.28 & 8.76 & 0.38 & 26.05 & 0.55 \\
\hline $\begin{array}{l}\text { NLOS } \\
\text { Enlace Pasillo-Oficina (B-E) } \\
\text { Puertas Cerradas }\end{array}$ & $\begin{array}{l}\text { Horizontal- } \\
\text { Vertical }\end{array}$ & 13.12 & 0.24 & 5.27 & 0.24 & 14.04 & 0.42 \\
\hline
\end{tabular}

